\title{
Oldenburg Burnout Inventory - validação de uma nova forma de mensurar Burnout no Brasil
}

\author{
Oldenburg Burnout Inventory - validation of a new way to measure \\ Burnout in Brazil
}

Marcelo da Silva Schuster ${ }^{1}$

Valéria da Veiga Dias ${ }^{2}$

\footnotetext{
${ }^{1}$ Universidade Federal de Santa Maria. Avenida Roraima 1000/74C, Camobi. 97050-330 Santa Maria RS Brasil.marcelo.schuster@ gmail.com ${ }_{2}^{2}$ Centro Universitário Franciscano. Santa Maria RS Brasil.
}

\begin{abstract}
This study sought to validate the Oldenburg Burnout Inventory (OLBI) measurement scale in Brazil. The methodology included data collection through a quantitative survey with 273 people. Structural equation modeling was used for data analysis. The scale was validated after being subjected to all statistical assumptions, reducing the original 16 to 13 variables resulting in an enhanced measurement capacity of the Burnout syndrome than the original scale. This study contributes a scale adapted to Brazil to study the health of the worker, since it provides a way to measure a syndrome responsible for problems of various types, with impacts both inside and outside organizations.
\end{abstract}

Key words OLBI, Burnout, Exhaustion, Switching off from work
Resumo Este estudo objetivou a validação da escala Oldenburg Burnout Inventory (OLBI) para o Brasil. A metodologia incluiu uma coleta de dados por meio de uma pesquisa quantitativa do tipo survey com 273 pessoas. Para a análise de dados foi utilizada a modelagem de equações estruturais. A escala foi validada após ser submetida a todos os pressupostos estatísticos, tendo sua estrutura reduzida de 16 para 13 variáveis, o que melhorou seu poder de mensuração. O presente estudo contribui com a literatura ao apresentar uma escala adaptada ao Brasil, que pode ser usada em estudos na área de saúde do trabalhador, uma vez que proporciona uma maneira de mensurar uma síndrome responsável por problemas de diversas ordens, com impactos dentro e fora das organizações.

Palavras-chave OLBI, Burnout, Exaustão, Desligamento do trabalho 


\section{Introdução}

Diante da dinâmica atual dos mercados, caracterizada pela elevada competitividade organizacional, o material humano configura-se como uma fonte de qualidade e diferencial competitivo nestas organizações. Em função disso, nota-se a emergência de novas rotinas de trabalho, competências essenciais e necessidade de envolvimento dos trabalhadores com seu local de trabalho, que muitas vezes levam o trabalhador ao limite da exaustão emocional e profissional. A dedicação em tempo integral ou a preocupação com aspectos do trabalho em tempo integral sinalizam o quanto é relevante a compreensão dos aspectos relacionados ao comportamento humano no contexto da saúde do trabalhador, tal como um diagnóstico de elementos estressores que podem comprometer resultados e a saúde individual.

A percepção dos trabalhadores sobre a discrepância entre seus esforços realizados e os objetivos alcançados no trabalho pode gerar uma série de sentimentos de frustração e estresse interpessoal. Quando a exposição aos elementos estressores e a frustração ocorrem por prolongado período de tempo, pode desencadear o desenvolvimento da síndrome de Burnout ${ }^{1}$.

Esta síndrome pode ser caracterizada como uma reação associada às condições de trabalho inicialmente identificadas em pessoas que desempenhavam funções com intensa interação humana, principalmente ligada à área da saúde ${ }^{2}$. Percebeu-se ao longo do tempo que a ocorrência da síndrome também está intimamente associada com ambientes onde o estresse, a frustração e a pressão no trabalho são uma constante, independente da função exercida ou área de trabalho ${ }^{3}$.

De acordo com Maroco e Tecedeiro ${ }^{4}$, a síndrome de Burnout reflete um sofrimento pessoal, manifestado por meio de indicadores psicossociais diversos como: consumo excessivo de medicamentos, álcool e outras substâncias psicotrópicas, afastamentos prolongados do trabalho por motivo de saúde, redução da produtividade, aumento do absentismo, episódios depressivos graves, perturbações psicossomáticas graves, intenção de desistir do trabalho e pouco comprometimento organizacional; ; além disso, outros indicadores relacionados a convivência social.

Em função de todas estas consequências da síndrome, diversos estudos anteriores procuraram estabelecer uma classificação das dimensões que fazem parte da síndrome e foram identificadas três dimensões essenciais: a exaustão, o cinismo e a eficácia profissional. No entanto, outros pesquisadores têm sugerido um modelo de dois fatores, que inclui apenas a exaustão emocional e despersonalização ${ }^{6}$. Esta identificação de fatores refletiu na criação de escalas e mensuração, dentre elas, as escalas mais referenciadas sobre o tema são a Maslach Burnout Inventory MBI, e a Oldenburg Burnout Inventory, ${ }^{6}$ que surgiu para suprir algumas limitações psicométricas da escala anterior, a MBI.

Apesar do esforço realizado para expandir os estudos sobre o Burnout no mundo, apenas nos últimos anos começou-se a estudá-la no Brasil. Em função disso, este estudo destaca os possíveis reflexos da síndrome na saúde das pessoas e organizações, ressaltando a importância da identificação da mesma em nossa realidade. Para isso, optou-se por realizar um estudo com o objetivo de validar a escala Burnout, Oldenburg Burnout Inventory (OLBI) no Brasil, e contribuir com o avanço dos estudos deste tema na realidade nacional.

O presente trabalho encontra-se estruturado nesta introdução, na conceituação sobre as formas de mensuração da Síndrome de Burnout, nos métodos utilizados e nos resultados encontrados na validação das escalas. Por fim, são apresentadas as considerações finais e as referencias utilizadas.

\section{Oldenburg Burnout Inventory (OLBI)}

O conceito da síndrome de Burnout é proveniente dos estudos de Freudenberger (1974, 1975) e Maslach (1976), ambos trabalhando de forma independente nos EUA, fornecendo a primeira descrição do fenômeno Burnout, e relacionando a ocorrência relativamente comum em trabalhos de assistência humana ${ }^{7}$.

Maslach et al. ${ }^{8}$ afirmam que os estudos de Burnout se desenvolveram em três fases, sendo a primeira a articulação do fenômeno por meio de entrevistas e observação de campo; a segunda fase, por meio da criação de escalas para a mensuração da síndrome; e a terceira, por meio de estudos longitudinais.

Elaboradas as construções teóricas na primeira fase, a segunda fase se desenvolveu na década de 1980, quando diversas escalas foram criadas, tais como: o Cuestionário de Burnout Del Profesorado (CBP) e Cuestionário Breve de Burnout (CBB) de Moreno-Jimenez et. al. (1997); a Copenhagen Burnout Inventory (CBI) de Kristensen et. al. (2005); Shirom-Melamed Burnout Measure (SMBM) de Shirom e Melamed (2006); Burnout Measure (BM) de Jones (1980); Escala de Carac- 
terização do Burnout de Tamayo e Trócoli (2009), a Oldenburg Burnout Inventory (OLBI) desenvolvido pela Demerouti em 1999 e a MBI de Cristina Maslach ${ }^{9}$.

Halbesleben e Demerouti ${ }^{10}$ afirmam que a medida mais comumente utilizada para a mensuração de Burnout é a Maslach Burnout Inventory, desenvolvida no início de 1980, por Maslach e Jackson (1981), baseada em um modelo de três fatores de Burnout, inclui dimensões para medir a exaustão emocional, despersonalização e realização pessoal.

Esta escala apresenta diversas versões: a versão original para Serviços Humanos (MBI-HSS); uma versão para trabalhadores ligados a área de educação (MBI-ES); uma versão para estudantes (MBI-SS); e uma versão desenvolvida, em 1996, para abranger todas as profissões (MBI-GS). Atualmente, já se sabe que a ocorrência da síndrome de Burnout não se restringe a determinadas profissões, isso porque os estressores podem estar presentes em qualquer local de trabalho ${ }^{11}$.

Demerouti et al. ${ }^{12}$ argumentam que, do ponto de vista psicométrico, a MBI-GS apresenta uma série de falhas, tal como o possível enviesamento das respostas, já que as questões são apresentadas no sentido negativo para as dimensões exaustão e cinismo e, no sentido positivo, para a dimensão eficácia no trabalho. Outra falha apontada pelos autores refere-se a dimensão exaustão, que mensura somente aspectos afetivos, ignorando aspectos físicos e cognitivos do trabalho.

A fim de superar essas críticas foi desenvolvido um instrumento alternativo para mensurar a síndrome de Burnout, a escala Oldenburg Burnout Inventory (OLBI), criada por Evangelia Demerouti, em 1999. Esta escala é formada por dois fatores: exaustão e desligamento do trabalho ${ }^{12}$, e pode ser aplicada em qualquer contexto ocupacional.

O OLBI é semelhante à MBI-GS, ambos os instrumentos foram formulados para refletir a conceituação de Burnout sem restrição às profissões, com perguntas que se aplicam a qualquer grupo ocupacional ${ }^{11}$. Na OLBI, a exaustão é definida como uma consequência da intensa pressão, afetiva e física, isto é, como uma consequência de longo prazo à certas exigências desfavoráveis de trabalho. A dimensão desligamento do trabalho refere-se a distanciar-se do objeto e conteúdo do trabalho, particularmente com respeito à identificação com o trabalho e vontade de continuar na mesma profissão ${ }^{13}$.

A OLBI foi estruturada a partir de duas subescalas, com 8 (oito) questões para mensurar a exaustão, e outras 8 (oito) questões para mensu- rar o desligamento do trabalho; sendo que quatro questões de cada dimensão foram formuladas no sentido positivo e outras quatro no sentido negativo. A escala utiliza a estrutura do tipo likert para respostas, variando de 1 (um) a 4(quatro), sendo 1(um) discordo plenamente e 4(quatro) concordo plenamente.

\section{Método}

A fim de alcançar os objetivos propostos, foi realizada uma pesquisa do tipo survey com uso de instrumento de coleta de dados estruturado e divulgação por meio online. Hair Junior et al. ${ }^{14}$ afirmam que cada autor sugere um tamanho de amostra mínima. No entanto, para a realização da análise de equações estruturais, é desejável uma amostragem mínima de 15 respondentes por variável (questão) do instrumento de coleta de dados. Em função da quantidade de questões da escala, seriam necessários 240 respondentes.

Não houve dados faltantes, beneficio proporcionado pela coleta de dados por meio de formulário eletrônico, que exige o preenchimento de todas as questões. Para a validação da escala, inicialmente foi realizada a validade de expressão, antes da aplicação da pesquisa de campo. Na sequência foi realizada a coleta de dados e análise por meio da técnica de equações estruturais. Segundo $\mathrm{Ho}^{15}$ este é o melhor método para a validação de uma escala porque é flexível e compreensível, já que analisa os padrões de relacionamento entre as variáveis observáveis e latentes em um único modelo de mensuração que pode ser avaliado estatisticamente.

Hair Junior et al. ${ }^{14}$ estabelecem que para a validação de uma escala é necessário obter-se o ajuste geral do modelo de mensuração e as validades convergentes, divergentes e unidimensionalidades. Schumacker e Lomax ${ }^{16}$ explicam que o ajuste do modelo é a determinação do grau em que a matriz de variância e covariância da amostra se ajusta ao modelo de equações estruturais.

Diversos autores ${ }^{14,17-19}$ explicam que existe uma variedade de índices para avaliação do modelo, classificados em Índices Absolutos que avaliam a qualidade do modelo, sem comparação com outros modelos ( $c^{2} ; c^{2} / g l$; Std RMR e GFI); Índices Relativos que avaliam a qualidade sob teste relativamente ao modelo com pior ajustamento (NFI; CFI; TLI) e Índices de Discrepância Populacional que avaliam se o modelo ajustado é aproximadamente correto (RMSEA), conforme detalhado no Quadro 1. 


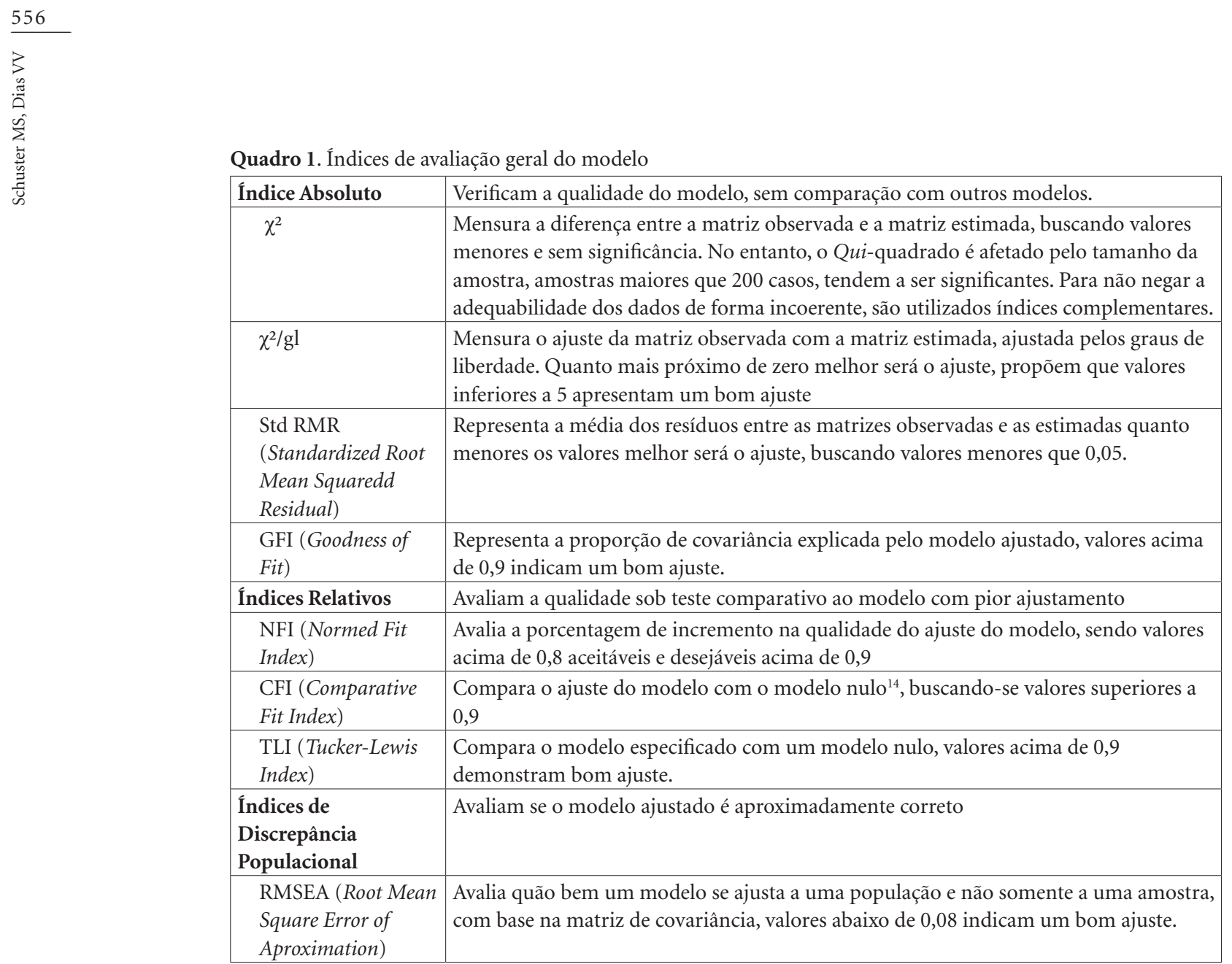

Fonte: adaptado de diversos autores ${ }^{14,15,18,19}$.

No que diz respeito às validades, a validade convergente é obtida pelo compartilhamento de uma elevada variância comum entre os itens do construto especifico, ou seja, as cargas fatoriais têm que que se apresentarem altas, devendo ser acima de 0,5 , estatisticamente significativas no teste T-student $(\mathrm{p}<0,001)$ e apresentando T-valor $>1,96)^{14}$.

A validade discriminante é calculada pela comparação entre a variância extraída do construto e suas variâncias compartilhadas (quadrado dos coeficientes de correlação) com os demais construtos, sendo necessário um valor maior da variância extraída para comprovação dessa validade $^{14}$. Já a unidimensionalidade é verificada por meio da matriz de covariância das variáveis, sendo que os valores desejados para os resíduos padronizados devem estar abaixo de $\left.|2,58|\right|^{14}$.

Além da validade do construto, também foi calculada a confiabilidade, que consiste em avaliar até que ponto uma escala produz resultados consistentes quando são feitas repetidas mensurações, ou seja, até que ponto a forma de mensuração está livre de erros aleatórios ${ }^{20}$. A confiabilidade foi mensurada pela confiabilidade composta, que Hair Junior et al. ${ }^{14}$ explicam ser a melhor forma de avaliar a confiabilidade em modelos de equações estruturais, sendo calculada por meio do somatório das cargas padronizadas ao quadrado dividido pelo somatório das cargas padronizadas ao quadrado mais o somatório do erro. Os resultados e discussões são apresentados na próxima seção.

\section{Resultados e discussão}

Visando a melhor compreensão da validação da escala Oldenburg Burnout Inventory (OLBI) na realidade brasileira, os resultados serão apresentados por etapas realizadas.

\section{Validade de expressão}

A primeira etapa de validação diz respeito à escala utilizada, Oldenburg Burnout Inventory 
(OLBI), que foi criada e validada por Evangelia Demerouti, na Alemanha, em 1999, em diferentes grupos ocupacionais e inclui dois fatores: exaustão e desligamento do trabalho ${ }^{12}$. A mesma autora, também validou a escala na língua ingle$\mathrm{sa}^{10}$, a versão traduzida para o inglês foi utilizada para realização deste estudo.

A OLBI é composta de 16 questões, mensuradas por meio de uma escala tipo likert de 4 pontos, variando de 1 (discordo plenamente) a 4 (concordo plenamente). Cada fator apresentou oito questões, quatro formuladas em sentido positivo e quatro no sentido negativo. Este instrumento pode ser utilizado em praticamente qualquer contexto ocupacional e sua construção visou superar uma importante lacuna psicométrica da MBI e MBI-GS em relação ao enviesamento das respostas, conforme anteriormente mencionado ${ }^{12}$.

Essa escala foi traduzida e validada em diversos idiomas, porém no português, e principalmente no Brasil, não foi encontrada referencia de sua validação. Em uma pesquisa na base de dados Scielo, foram encontrados somente dois estudos uma versão para estudantes, e uma versão para dentistas ${ }^{21}$.

Sendo assim, primeiro foi realizada a tradução do inglês para o português por dois tradutores independentes e qualificados que estavam cientes dos objetivos da tradução, na sequência foi realizada a tradução reversa para o inglês ( $b a-$ ck-translation) por outro tradutor independente e bilíngue. Outra etapa foi a revisão da escala por um grupo de pesquisadores que visou produzir uma versão final da escala, comparando os resultados entre si. Após os procedimentos de tradução e análise dos itens, o instrumento ficou estruturado conforme Quadro 2 e foi então aplicado à amostra do estudo.

\section{Caracterização da amostra de pesquisa}

O total de respondentes nesse estudo foi de 273 pessoas. Deste total, 96 pessoas são do gênero masculino, representando $35 \%$ do total e 177 do gênero feminino, representando $65 \%$ do total. Em função de que existiu diferença entre a quantidade de respondentes do gênero masculino e feminino na amostra, foi realizado o teste de invariância com a finalidade de verificar se o comportamento das variáveis é diferente considerando o gênero. O teste de Levene ${ }^{14}$, realizado no software SPSS indicou que não há diferença no comportamento dos dados para o gênero, portanto, não é necessária a diferenciação entre os resultados de homens e mulheres.

No que tange ao estado civil, prevalecem às pessoas casadas (143), que representam $52 \%$ do total, seguido de solteiros (110) e separados/divorciados/viúvos (20). Em relação a escolarida-

Quadro 2. Variaveis por fator de Burnout.

\begin{tabular}{|l|c|}
\hline & Fator \\
\hline Há dias em que me sinto cansado antes mesmo de chegar ao trabalho & EE1 \\
\hline Depois do trabalho, preciso de mais tempo para sentir-me melhor do que precisava antigamente & EE2 \\
\hline Consigo suportar muito bem as pressões do meu trabalho & EE3 \\
\hline Durante o meu trabalho, sinto-me emocionalmente esgotado & EE4 \\
\hline Depois das tarefas profissionais, tenho energia para as minhas atividades de lazer & EE5 \\
\hline Quando trabalho, sinto-me bem & EE6 \\
\hline Depois do trabalho, sinto-me cansado e sem energia & EE7 \\
\hline De uma forma geral, consigo administrar bem a quantidade de trabalho que tenho & EE8 \\
\hline Com frequência faço coisas novas e interessantes no meu trabalho & DT1 \\
\hline Cada vez falo mais e com mais frequência de forma negativa sobre meu trabalho & DT2 \\
\hline Ultimamente, tenho realizado meu trabalho de forma quase mecânica & DT3 \\
\hline Considero meu trabalho um desafio positivo & DT4 \\
\hline Com o passar do tempo, venho me desinteressado do meu trabalho & DT5 \\
\hline O trabalho que faço hoje é o único que me imagino fazendo & DT6 \\
\hline Sinto-me cada vez mais empenhado no meu trabalho & DT7 \\
\hline Muitas vezes sinto-me farto das minhas tarefas & DT8 \\
\hline
\end{tabular}


de, a maior parte dos respondentes (82\%) possui alta escolaridade, ou seja, pós-graduação (143) e graduação (82); em seguida, com $16 \%$ do total aparecem os respondentes com ensino médio e profissionalizante (44) e $11 \%$ possuem ensino médio e fundamental (31). A faixa etária de mais presente na amostra a de 26 a 35 anos (50\%), seguido de 36 a $45(19 \%), 17$ a $25(16 \%), 45$ a 55 (11\%) e 56 a 66 (4\%).

O perfil dos respondentes referente a atividade desempenhada revelou que parte da amostra estuda e desempenha as atividades laborais (58,5\%). Quanto ao setor de trabalho, o setor de serviços públicos foi o que mais apareceu $(42,6 \%)$, seguido de serviços $(39,9 \%)$, comércio $(9,5 \%)$ e indústria (8\%). Desta amostra 34,8\% exerce funções de chefia.

Para avaliação das médias dos construtos, exaustão e Desligamento do Trabalho foram invertidos os valores das seguintes variaveis $\mathrm{EE} 3$; EE5; EE6; EE8; DT1; DT4; DT6 e DT8, caracterizando-se assim quanto maior a média, maiores os índices de Burnout. No presente estudo, as médias de exaustão encontradas foram 2,33 (desvio padrão de 0,45$)$ e desligamento do trabalho a média foi 2,4 (desvio padrão de 0,51). Em uma escala com variação de 1 a 4 , estes valores podem ser classificados como dentro de patamares intermediários, ou seja, a população da pesquisa apresenta níveis de Burnout que demandam atenção.

\section{Validação do modelo de mensuração}

Verificou-se que nesta amostra nenhuma variável apresentou valores superiores aos indicados, comprovando a normalidade multivariada desses. A análise da normalidade multivariada foi realizada por meio do software IBM SPSS AMOS $^{\circledR}$. Kline ${ }^{17}$ propõe como referência de análise da normalidade, a simetria e a curtose, onde valores absolutos da simetria $<3$ e curtose $<8$ não apresentarão problema para a análise dos dados.

Para Hair Junior et al. ${ }^{14}$ a validade do modelo de mensuração depende da qualidade do mesmo, esta qualidade especifica quão bem o modelo de mensuração reproduz a matriz de covariância entre os indicadores. A fim de mensurar a qualidade do modelo, inicialmente foi alterado o sentido das variáveis EE1, EE2, EE4, EE7, DT2, DT3, DT5, DT8, e os resultados indicam que, quanto maior o índice, menor é o nível de Burnout. Dessa maneira não seria encontrada variância negativa entre as variáveis do mesmo fator, facilitando a execução e análise dos testes no software AMOS.
A avaliação do modelo inicial apresentou um $c^{2}$ (diferença entre a matriz observada e a matriz estimada, buscando-se valores menores e sem significância,) de 247, 338 com 103 graus de liberdade e significância $(\mathrm{p}<0,0001)$, o que pode ter ocorrido devido ao tamanho da amostra. $\mathrm{Ho}^{15}$ explica que amostras com mais de 200 respondentes apresentam propensão maior a apresentar significância no teste de $\mathrm{c}^{2}$.

Para não negar o modelo de forma incoerente, foram utilizados índices absolutos como o $\mathrm{c}^{2} / \mathrm{gl}$ $(2,4)$ conhecido como qui-quadrado relativo, que busca realizar a análise diminuindo a influencia do tamanho da amostra, onde valores menores que 5 indicam um bom auto ajuste, o GFI que explica a proporção de covariância explicada pelo modelo ajustado apresentou um valor de 0,91, onde valores acima de 0,9 são desejáveis.

Índices Relativos avaliam a qualidade sob testes relativos ao modelo com pior ajustamento, tais como o NFI, que avalia a porcentagem de incremento na qualidade do ajuste do modelo, foi 0,87 ; TLI, que compara o modelo especificado com um modelo nulo, foi 0,91 ; CFI, que compara o ajuste do modelo, foi 0,92 , todos esses buscando valores acima de $0,9^{14,15,17,19}$.

Índices de Discrepância Populacional avaliaram se o modelo ajustado é aproximadamente correto, usando o RMSEA, que representa o quão bem um modelo se ajusta a uma população e não somente a uma amostra, apresentou valor de 0,07 , e o Std RMR, que corresponde a uma média dos resíduos entre as matrizes observadas e estimadas, foi de 0,06. Quanto menores esses índices, melhor é a avaliação da escala ${ }^{14}$. A escala apresentou um bom ajuste, somente alguns índices ficaram abaixo do desejado.

O próximo passo foi à verificação da validade convergente. Essa não foi comprovada, pois algumas variáveis apresentaram cargas padronizadas menores que 0,5 , sendo assim, optou-se pela análise e exclusão das mesmas em sequência como segue descrito. A primeira foi a variável DT6 $(O$ trabalho que faço hoje é o único que me imagino fazendo), que apresentou uma carga de 0,26 , além de um poder de mensuração de menos de $7 \%$, ou seja, sua contribuição é muito baixa para a mensuração do desligamento do trabalho. A seguir foi excluída a variável EE8 (De uma forma geral, consigo administrar bem a quantidade de trabalho que tenho), que apresentou uma carga de $0,45 \mathrm{e}$ um poder de mensuração abaixo de $20 \%$, o que pode ser proveniente da tradução da escala, interpretação dos respondentes ou até mesmo por questões culturais, uma vez que essa escala foi 
criada na Alemanha e as condições de trabalho são diferentes no país.

Com esses procedimentos foi alcançada a validade convergente, ou seja, variáveis com valores significativos no teste T-student ( $\mathrm{p}<0,001)$, $\mathrm{T}$ valor $>1,96$ e cargas padronizadas maiores que 0,5 . Também foi realizada a verificação da unidimensionalidade da escala, por meio dos resíduos padronizados de covariância, sendo necessário que não haja covariância maior que $|2,58|$. Este fato foi verificado, comprovando assim a unidimensionalidade do modelo.

As últimas análises dizem respeito à confiabilidade do construto e a validade discriminante. A confiabilidade foi mensurada pela validade composta, valores acima de 0,7 são ideais. Nesta escala a dimensão exaustão emocional apresentou confiabilidade de 0,92 e a dimensão desligamento do trabalho uma confiabilidade de 0,88 , o que significa que as duas dimensões possuem ótima confiabilidade. No que tange a validade discriminante (alcançada quando a variância extraída média (VEM) dos fatores for maior que o quadrado das correlações apresentadas por esse), não foi possível obtê-la, isso porque a exaustão emocional apresentou a VEM abaixo do quadrado das correlações entre os fatores $(0,54)$. Com isso, ficou demonstrado que a escala apresenta uma boa confiabilidade, mas sem uma grande distinção entre os fatores, como pode ser verificado na Tabela 1.

O problema encontrado na validade discriminante pode ser decorrente do baixo poder de mensuração de algumas variáveis, o que segundo Hair Junior et al. ${ }^{14}$ pode ter relação com a ligações das variáveis com outros construtos, apresentando assim uma VEM próxima do valor das correlações quadráticas.

$\mathrm{Na}$ avaliação global, a escala apresentou uma ótima confiabilidade e um ótimo ajuste do modelo de mensuração, apresentando a validade convergente e unidimensional. O único desajuste ocorreu na validade divergente, que pode ter ocorrido em função do ambiente onde a escala foi desenvolvida, ou algum problema de adaptação à realidade nacional.

Considerando o caráter exploratório deste estudo, buscou-se verificar através dos índices de modificação do software AMOS qual seria o melhor ajuste da escala, procedendo com exclusões de variáveis que apresentam desajustes de mensuração. Para a realização deste procedimento foram analisados os índices de modificação apresentados pelo software, que apresenta as relações que melhorariam o modelo de mensuração, além das correlações múltiplas quadradas, que Hair

Tabela 1. Índices de Validação OLBI.

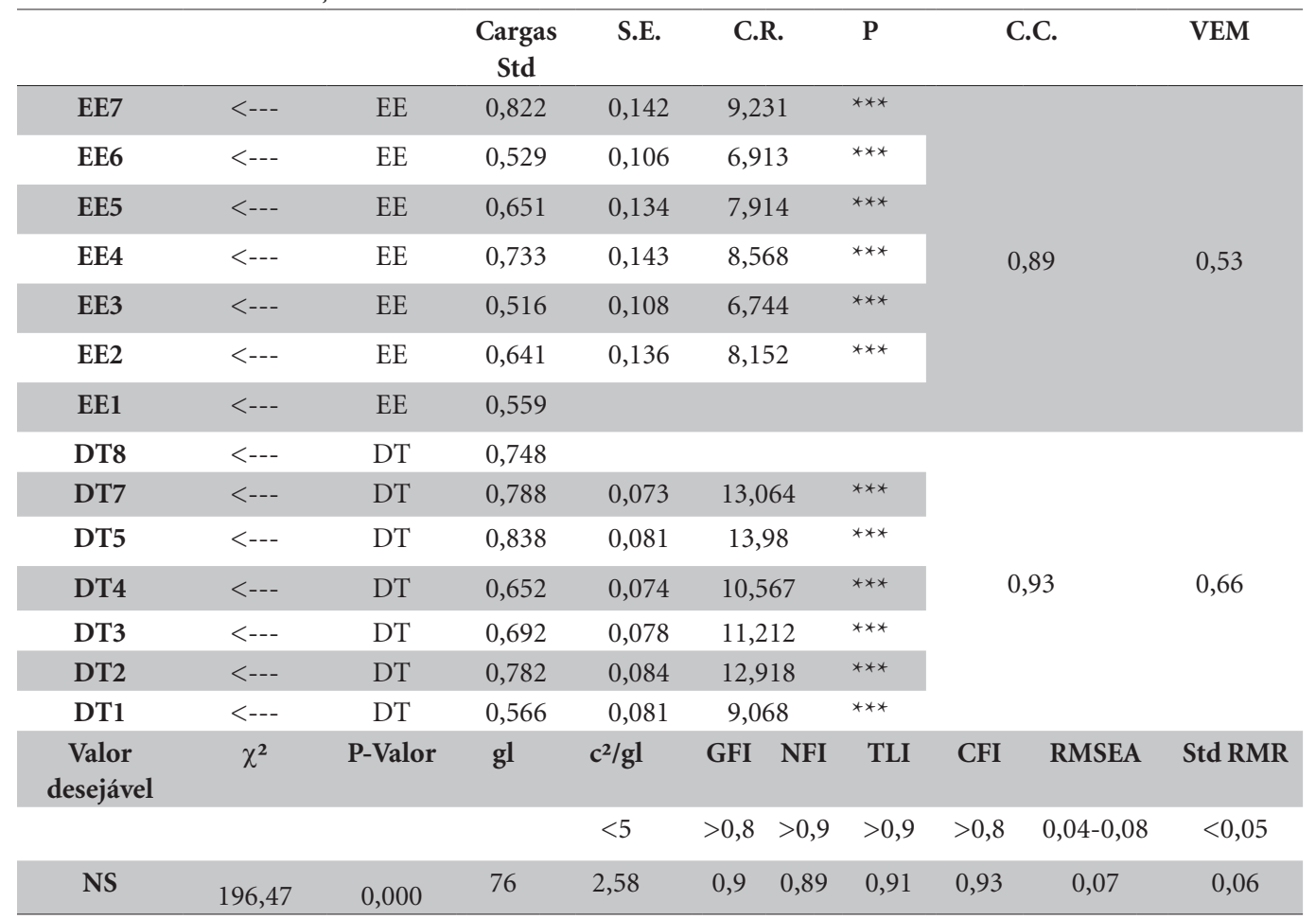


Junior et al. ${ }^{14}$ afirmam demonstrar o poder de mensuração do item com a escala.

A variável EE6, apresentou um baixo poder de mensuração (20\%) e uma correlação com o fator desligamento do trabalho, podendo ser um dos motivos de comprometimento da validade divergente. Portanto, foi excluída. Com a exclusão dessa variável, o modelo passou a apresentar índices de ajuste melhores e alcançou a validade convergente, conforme é apresentado na Tabela 2.

O modelo da Oldenburg Burnout Inventory apresentou alguns ajustes, conforme pôde ser verificado na Tabela 2. Hair Junior et al. ${ }^{14}$, Costa $^{22}$ e Maroco ${ }^{23}$ afirmam existir uma diversidade de índices de ajustes do modelo de equações estruturais, neste estudo buscou-se o uso do maior número de índices que fossem complementares com a intenção de tornar o modelo melhor.

\section{Modelo validado}

A escala Oldenburg Burnout Inventory, inicialmente apresentou duas dimensões com oito variáveis cada. Após a realização do procedimento de validação da escala e com a exclusão de três questões que apresentaram problemas no poder de mensuração, o modelo exibiu um melhor ajuste, além de alcançar as validades convergente, divergente e unidimensionalidade.

Byrne $^{19}$ afirma que, para avaliar a qualidade do modelo inicial e final, se deve proceder com o cálculo da diferença entre os valores do $\chi^{2}, \chi^{2} /$ df e ECVI, este último para ver a melhor adequação à amostra. Neste estudo os valores do modelo apresentaram melhora e o modelo final com um melhor ajuste à amostra pesquisada, conforme apresentado na Tabela 3.

A diferença entre o inicial e o final comprova a melhoria do modelo, indicando o melhor ajustamento em outras amostras. O Índice ECVI apresentou um valor final de 0,795 , demonstrando um ótimo ajuste da escala. Segundo $\mathrm{Maroco}^{23}$, quanto menor o valor do ECVI, melhor será a adequação.

A escala ficou estruturada em seis variáveis (questões), para a verificação da exaustão, sendo que duas questões foram formuladas no sentido positivo e quatro no sentido negativo. Para o desligamento do trabalho restaram sete variáveis (questões), sendo três formuladas no sentido positivo e quatro no sentido negativo, conforme demonstra a Tabela 4.

Com treze questões, a escala apresentou uma melhoria em relação ao modelo proposto pela $O L B I$ original e o primeiro modelo apresentado nesse artigo. Essa escala apresentou uma melhora para o tema já que conseguiu, com ótima confiabilidade e ajuste do modelo de mensuração, medir os mesmos construtos com menor número de questões e maior poder de mensuração da relação entre o estresse e o trabalho. Ressalta-se que não foram encontrados índices de ajuste do modelo confirmatório para esta escala em outros estudos, para que se fizesse uma comparação com os achados desta pesquisa.

Tabela 2. Índices de validação OLBI ajustada.

\begin{tabular}{|c|c|c|c|c|c|c|c|c|c|c|}
\hline & & & Cargas Std & S.E. & & C.R. & $\mathbf{P}$ & C. & & VEM \\
\hline EE7 & $<---$ & $\mathrm{EE}$ & 0,84 & 0,144 & & 9,283 & $* * *$ & \multirow{6}{*}{\multicolumn{2}{|c|}{0,78}} & \multirow{6}{*}{0,57} \\
\hline EE5 & $<---$ & $\mathrm{EE}$ & 0,669 & 0,136 & & 8,025 & $* * *$ & & & \\
\hline EE4 & $<---$ & $\mathrm{EE}$ & 0,721 & 0,141 & & 8,493 & $* * *$ & & & \\
\hline EE3 & $<---$ & $\mathrm{EE}$ & 0,515 & 0,108 & & 6,72 & $* * *$ & & & \\
\hline EE2 & $<---$ & $\mathrm{EE}$ & 0,643 & 0,135 & & 8,167 & $* * *$ & & & \\
\hline EE1 & $<---$ & $\mathrm{EE}$ & 0,561 & & & & & & & \\
\hline DT8 & $<---$ & DT & 0,749 & & & & & \multirow{7}{*}{0,88} & & \multirow{7}{*}{0,66} \\
\hline DT7 & $<---$ & DT & 0,788 & 0,073 & & 13,04 & $* * *$ & & & \\
\hline DT5 & $<---$ & DT & 0,84 & 0,081 & & 13,997 & $* * *$ & & & \\
\hline DT4 & $<---$ & DT & 0,654 & 0,074 & & 10,578 & $* * *$ & & & \\
\hline DT3 & $<---$ & DT & 0,691 & 0,078 & & 11,2 & $* * *$ & & & \\
\hline DT2 & $<---$ & DT & 0,781 & 0,084 & & 12,878 & $* * *$ & & & \\
\hline DT1 & $<---$ & DT & 0,565 & 0,081 & & 9,053 & $* * *$ & & & \\
\hline \multirow[t]{2}{*}{$\begin{array}{c}\text { Valor } \\
\text { desejável }\end{array}$} & \multicolumn{2}{|c|}{$c^{2}$} & P-Valor & $c^{2} / g l$ & GFI & NFI & TLI & CFI & RMSEA & $\begin{array}{c}\text { Std } \\
\text { RMR }\end{array}$ \\
\hline & \multirow{2}{*}{\multicolumn{2}{|c|}{154,031}} & & $<5$ & $>0,8$ & $>0,9$ & $>0,9$ & $>0,8$ & $0,04-0,08$ & $<0,05$ \\
\hline NS & & & 0,000 & 2,407 & 0,92 & 0,91 & 0,93 & 0,94 & 0,07 & 0,05 \\
\hline
\end{tabular}




\section{Considerações finais}

A Oldenburg Burnout Inventory (OLBI) foi desenvolvida em 1999, no idioma alemão, traduzida para o inglês e, posteriormente, validada para diversos idiomas. No entanto, não foi encontrada referência de sua validação para o português, ou para uso em estudos no Brasil. Sendo assim, este estudo se propôs a realização da validação da OLBI, para a realidade do Brasil.

A escala demonstrou um bom ajuste à amostra nacional, apresentando bons índices de ajuste global. As validades convergentes, divergente e unidimensional foram alcançadas com a exclusão de três variáveis, o que pode ser explicado por questões de tradução, interpretação e até mesmo culturais, uma vez que os ambientes e sistemas onde a escala foi desenvolvida diferem do brasileiro.

O modelo final da escala foi estruturado com treze variáveis, o que conferiu uma melhoria significativa do ajuste dos índices em comparação ao modelo inicial. Este estudo representa uma contribuição para a literatura nacional sobre a síndrome de Burnout, já que descreve e analisa de

Tabela 3. Diferença entre modelo inicial e final da OLBI.

\begin{tabular}{lrrr}
\hline & \multicolumn{1}{c}{$\chi^{2}$} & $\chi^{2} / \mathrm{DF}$ & ECVI \\
\hline Modelo Inicial & 196,47 & 2,58 & 0,936 \\
Modelo Final & 154,031 & 2,407 & 0,765 \\
$\Delta$ & 42,439 & 0,173 & 0,171 \\
\hline
\end{tabular}

forma detalhada as etapas de validação de uma das escalas mais usadas para a mensuração deste fenômeno; o estudo ainda colabora no sentido de realizar a pesquisa em população geral sem restrição de cargos. A validação, bem como a aplicação da escala sem restrição na realidade do Brasil, confere a este estudo um caráter inédito. Com a replicação da mesma e a validação transcultural, a mesma pode trazer importante contribuição para a literatura sobre o tema.

Além disso, este artigo traz contribuições quando abre uma janela de novas oportunidades para estudos para a área de saúde do trabalhador, uma vez que proporciona uma maneira de mensurar uma síndrome responsável por problemas de diversas ordens, com impactos dentro e fora das organizações.

Para futuras pesquisas, fica a sugestão de aplicação da escala validada em outros contextos, visando o aprimoramento dos instrumentos de mensuração, bem como do conhecimento da realidade sobre o tema no Brasil. Além disso, outros construtos que interferem na saúde do trabalhador podem ser explorados em modelos estruturados, tais como: justiça organizacional, satisfação com o trabalho, comportamento de cidadania organizacional, motivação, compromentimento entre outros que possam averiguar a influência do estado de esgotamento e desinteresse pelo trabalho nos objetivos organizacionais. No que tange especificamente à escala inicial, surgere-se a aplicação reavaliando a validade de expressão, por meio da inversão do sentido das questões, a fim de averiguar se o modelo resultaria em uma validação diferente da encontrada neste estudo.

Tabela 4. Modelo Final OLBI.

\begin{tabular}{lc}
\hline \multicolumn{1}{c}{ Variáveis } & Fator \\
\hline Com frequência faço coisas novas e interessantes no meu trabalho & DT1 \\
Cada vez falo mais e com mais frequência de forma negativa sobre meu trabalho & DT2 \\
Ultimamente, tenho realizado meu trabalho de forma quase mecânica & DT3 \\
Considero meu trabalho um desafio positivo & DT4 \\
Com o passar do tempo, venho me desinteressado do meu trabalho & DT5 \\
Sinto-me cada vez mais empenhado no meu trabalho & DT7 \\
Muitas vezes sinto-me farto das minhas tarefas & DT8 \\
Há dias em que me sinto cansado antes mesmo de chegar ao trabalho & EE1 \\
Depois do trabalho, preciso de mais tempo para sentir-me melhor do que precisava antigamente & EE2 \\
Consigo suportar muito bem as pressões do meu trabalho & EE3 \\
Durante o meu trabalho, sinto-me emocionalmente esgotado & EE4 \\
Depois das tarefas profissionais, tenho energia para as minhas atividades de lazer & EE5 \\
Depois do trabalho, sinto-me cansado e sem energia & EE7 \\
\hline
\end{tabular}




\section{Colaboradores}

MS Schuster trabalhou na pesquisa, estruturação do artigo e análises estatísticas e dos resultados. VV Dias trabalhou na escrita e conferência do texto, além de colaborar na coleta.

\section{Referências}

1. Vicente CS, Oliveira RA, Maroco J. Análise fatorial do Inventário de Burnout de Maslach (MBI-HSS) em profissionais portugueses. Psicologia, Saúde \& Doenças 2013; 14(1)152-167.

2. Dahlin M, Fjell J, Runeson, B. Factors at medical school and work related to exhaustion among physicians in their first postgraduate year. Nordic Journal of Psychiatry 2010; 64(6):402-408.

3. Pires DA, Monteiro AP, Alencar DR. Síndrome de Burnout em professores de Educação Física da região Nordeste do Pará. Pensar a Prática 2012; 15(4):821-1113.

4. Maroco, J, Tecedeiro, M. Inventário de Burnout de Maslach para estudantes portugueses. Psicologia, Saúde \& Doenças 2009; (10):227-235.

5. Mallmann CS, Palazzo LS, Carlotto MS, DRGC Aerts. Fatores associados à síndrome de Burnout em funcionários públicos municipais. Psicologia: teoria e prática 2009; 11(2):69-82.

6. Halbesleben JRB, Buckley MR. Burnout in organizational life. Journal of Management 2004; 30(6):859-879

7. Cox T, Tisserand M, Tariz T. The conceptualization and measurement of Burnout: Questions and directions. Work \& Stress 2005; 19(3):187-191.

8. Maslach C, Schaufeli WB, Leiter MP. Job Burnout. Annual Review of Psychology 2001; (52):397-422.

9. Tamayo MR, Tróccoli BT. Construção e validação fatorial da Escala de Caracterização do Burnout (ECB) Estudos de Psicologia 2009; 14:213-221.

10. Halbesleben JRB, Demerouti E. The construct validity of an alternative measure of Burnout: Investigating the English translation of the Oldenburg Burnout Inventory. Work and Stress 2005; 19(3):208-220.

11. Demerouti E, Bakker AB, Nachreiner F, Schaufeli WB. The job demands-resources model of Burnout. J App Psychol 2001; 86(3):499-512.

12. Demerouti E, Bakker AB, Vardakou I, Kantas A. The convergent validity of two Burnout instruments - A multitrait-multimethod analysis. European Journal of Psychological Assessment 2003; 19(1):12-23.

13. Demerouti E, Mostert K, Bakker AB. Burnout and Work Engagement: A Thorough Investigation of the Independency of Both Constructs. Journal of Occupational Health Psychology 2010; 15(3):209-222.
14. Hair Junior JF, Black WC, Babin BJ, Anderson RE, Tatham RL. Análise multivariada de dados. $6^{\mathrm{a}}$ ed. Porto Alegre: Bookman; 2009.

15. Ho R. Handbook of Univariate and Multivariate Data Analysis with IBM SPSS. Boca Raton: CRC Press; 2013.

16. Schumacker RE, Lomax RG. A beginner's guide to structural equation modeling. London: Routledge Academic; 2012.

17. Kline RB. Principles and practice of structural equation modeling. New York: Guilford press; 2011.

18. Meyers LS, Gamst G, Guarino AJ. Applied Multivariate Research: Design and Interpretation. Thousand Oaks: SAGE Publications; 2012.

19. Byrne BM. Structural equation modeling with AMOS: Basic concepts, applications, and programming. London: Routledge; 2013.

20. Malhotra NK. Pesquisa de Marketing: uma orientação aplicada. Porto Alegre: Bookman; 2012.

21. Campos JADB, Trotta OST, Bonafé FSS, Maroco J. Burnout em dentistas do serviço público - ter ou não ter, eis a questão! Revista de Odontologia da UNESP 2009; 39(2):109-114.

22. Costa F. Mensuração e desenvolvimento de escalas: aplicações em administração. Rio de Janeiro: Editora Ciência Moderna; 2011.

23. Maroco J. Análise de Equações Estruturais: Fundamentos teóricos, software \& Aplicações. Pêro Pinheiro: ReportNumber; 2010.

Artigo apresentado em 30/04/2015

Aprovado em 07/05/2016

Versão final apresentada em 09/05/2016 